\title{
Erratum to: Efficacy and safety of bisoprolol fumarate compared with carvedilol in Japanese patients with chronic heart failure: results of the randomized, controlled, double-blind, Multistep Administration of bisoprolol IN Chronic Heart Failure II (MAIN-CHF II) study
}

\author{
Masatsugu Hori - Ryozo Nagai - Tohru Izumi • \\ Masunori Matsuzaki
}

Published online: 19 June 2013

(C) Springer Japan 2013

Erratum to: Heart Vessels

DOI 10.1007/s00380-013-0340-3

Unfortunately, the below note was not included in the original publication.

"Masatsugu Hori, Ryozo Nagai and Tohru Izumi received lecture fees from Mitsubishi Tanabe Pharma Corporation".

The online version of the original article can be found under doi: 10.1007/s00380-013-0340-3.

M. Hori $(\bowtie)$

Osaka Medical Center for Cancer and Cardiovascular Diseases,

1-3-3 Nakamichi, Higashinari-ku, Osaka 537-8511, Japan

e-mail: hori-ma@mc.pref.osaka.jp

\section{R. Nagai}

Department of Cardiovascular Medicine, Graduate

School of Medicine, The University of Tokyo,

7-3-1 Hongo, Bunkyo-ku, Tokyo 113-8655, Japan

\section{T. Izumi}

Department of Cardio-Angiology, Kitasato University

School of Medicine, 1-15-1 Kitasato, Minami-ku,

Sagamihara, Kanagawa 252-0374, Japan

\section{Matsuzaki}

Department of Medicine and Clinical Science,

Division of Cardiology, Yamaguchi University Graduate

School of Medicine, 1-1-1 Minamikogushi, Ube,

Yamaguchi 755-8505, Japan 